\title{
Alterations in Aggregation Parameters of Erythrocytes Due to Hyper Cholesterol in Type-2 Diabetes Mellitus
}

\author{
N. Babu* \\ Department of Bio-engineering, SASTRA University, Thanjavur - 613402, Tamil nadu, India
}

\begin{abstract}
The aggregation parameters of erythrocytes in hyperglycemic subjects with normal cholesterol and with hyper cholesterol were analyzed, and compared with control, by optical scattering under dynamic conditions. Aggregation of erythrocytes is determined by online erythrocyte aggregometer, based on sequential analysis of transmitted laser light intensity after passing through the erythrocyte suspension. The aggregation mechanism in terms of various parameters like process initiation time (PIT), process completion time (PCT), aggregation size index (ASI), effective number of cells (ENC), and effective cellular sedimentation duration (ECSD) is determined. The aggregation parameters thus obtained are used to describe quantitatively the dynamic nature of aggregation process under gravitational sedimentation. The present data show that, in diabetes mellitus with normal cholesterol, the aggregation of erythrocytes is increased and in diabetes with hyper cholesterol, the aggregation is further increased significantly. These changes may significantly affect the flow properties of erythrocytes in microcirculation in diabetes mellitus with hyper cholesterol.
\end{abstract}

Keywords: Diabetes mellitus, erythrocyte aggregation, hypercholesterolemia, hyperglycemia.

\section{INTRODUCTION}

Erythrocytes in normal human blood tend to form aggregates under low shear-flowing conditions, which form face-to face morphology similar to a stack of coins called "rouleaux". Aggregation of erythrocytes is a result of the interaction of the erythrocyte membrane and plasma proteins such as fibrinogen and globulins [1]. Increased RBC aggregation has been observed in various pathological diseases, such as diabetes, hyper-cholesterol, thrombosis, myocardial infarction, vascular diseases, and hematological pathology. The major cause of aggregation is the presence of large plasma-proteins, especially fibrinogen $[2,3]$.

Diabetes mellitus is a disease associated with abnormal carbohydrate metabolism, arising from insulin deficiency and/or malfunction of insulin receptors [4]. 20-40\% elevation of fibrinogen has been detected in diabetic blood. Greater red cell aggregation is the major cause of increased low shear rate blood viscosity and elevated erythrocyte sedimentation rate. It is produced by high fibrinogen and heptaglobin levels that are cross sectionally linked to increased viscosity and to diabetic complications [5].

Red blood cells of diabetic patients have an altered membrane phospholipid asymmetry which influences the adhesion of erythrocytes to endothelial cells; it is also involved in red cell aggregation by altering the ability of erythrocytes to deform into rouleaux, particularly through a modification in membrane lipid fluidity [6].

Red cells from diabetic patients aggregate more readily than those from normal subject [7]. Erythrocyte aggregation

\footnotetext{
*Address correspondence to this author at the Department of Bioengineering, SASTRA University, Thanjavur - 613402, Tamil nadu, India; E-mail: santhurbabu@yahoo.co.in
}

in diabetes is favored by reduced negative surface charge. The surface sialic acid is responsible for $80 \%$ of the charge. This decrease has been linked to degree of hyperglycemia [8].

Hypercholesterolemia process also induces changes in the erythrocyte membrane. This is primarily attributed to accumulation of cholesterol in the membrane [9]. The erythrocyte membrane consists of two domains, the lipid bilayer and the cytoskeleton [10]. Phospholipids and cholesterol compose most of the lipid bilayer. Cholesterol is distributed evenly throughout the lipid domain, which alters flexibility and provides stability to the membrane. Any changes in cholesterol content of erythrocyte membrane changes the membrane property that alters the normal hemorheological properties like aggregation, deformability, shape etc. The aggregation, deformability and shape of the erythrocytes alter in diabetes mellitus [11-13] as well as in hyper cholesterol subjects $[14,15]$. The combined effect of both glucose and cholesterol makes complex changes in hemorheological parameters [15]. There are several in vivo mechanisms, which induce changes in erythrocyte membrane lipids and proteins as well as in intracellular viscosity, leading to alteration in erythrocyte shape, which in turn affects the deformability [16-20].

These studies show that hyperglycemia and hypercholesterolemia affect the hemorheological and morphological characteristics of erythrocytes. The objective of the present work is to determine the aggregation parameters of erythrocytes in blood samples of diabetic patients, with normal-cholesterol and hyper-cholesterol concentration, and to compare with healthy subjects in order to study the influence of cholesterol in diabetes mellitus on aggregation of erythrocytes. The technique has been used to measure the aggregation in various conditions like malaria [21], jaundice [22] and blood storage [23]. 


\section{MATERIALS AND METHODS}

\subsection{Online Erythrocyte Aggregometer}

This is based on the principles of the attenuation of transmitted intensity of laser light due to scattering by erythrocytes and their aggregates, on passing through the specimen chamber containing erythrocyte suspension in plasma at 5\% hematocrit in the path of the beam. Fig. (1) shows a schematic diagram of the system used for sequential analysis of aggregation process of erythrocytes. A light beam of He-Ne laser (power $=2 \mathrm{mw}$; Spectra Physics, USA) of wavelength $632.8 \mathrm{~nm}$ and diameter $1 \mathrm{~mm}$ was allowed to pass through the observed volume (OV) located at the center of the specimen chamber. The transmitted intensity (TI) was detected by a photo diode amplifier (HAD 1000A; EG \&G, USA). This output was fed to a 12-bit A/D converter, which was interfaced to a personal computer.

The specimen chamber, made up of optically flat glass plate having internal dimensions $70 \times 8 \times 1.6 \mathrm{~mm}$, was filled with erythrocyte suspension in plasma at $5 \%$ hematocrit. The chamber width was kept sufficiently large to allow interaction between cells and plasma proteins to form aggregates and for their movement away from the walls. Further details of the experimental technique have already been reported [11, 12].

The TI is associated with fluctuation characterized by peaks and troughs. Analysis of TI for Nt at the peak of a given fluctuation provides minimum number of cells (Ntmin) and at the trough provides the maximum number of cells (Ntmax). The variation in the number of cells (Nt max$\mathrm{Nt}$ min) is indicative of the change in aggregate size. To minimize the contribution of noisy fluctuations to the results, change in $(\mathrm{Nt} \max -\mathrm{Nt} \min )$ is thresholded at 3000 and remaining fluctuations are classified into four categories of various sizes based on the TI data's. The categories are the aggregates having the cells of (3000-3500), (3501-5000), (5001-10,000), and above 10,000.

\subsection{Aggregation Parameters}

Process initiation time (PIT) is the time in minutes taken for the appearance of fluctuation in TI and Process completion time (PCT) is the time required for the completion of the sedimentation till the TI reaches the intensity $\left(\mathrm{I}_{0}\right)$ when plasma alone in the OV. Aggregation size index (ASI) indicates the instantaneous change in the size of the aggregates given by the expression $\mathrm{Nt} \max _{\max }-\mathrm{Nt} \min$.

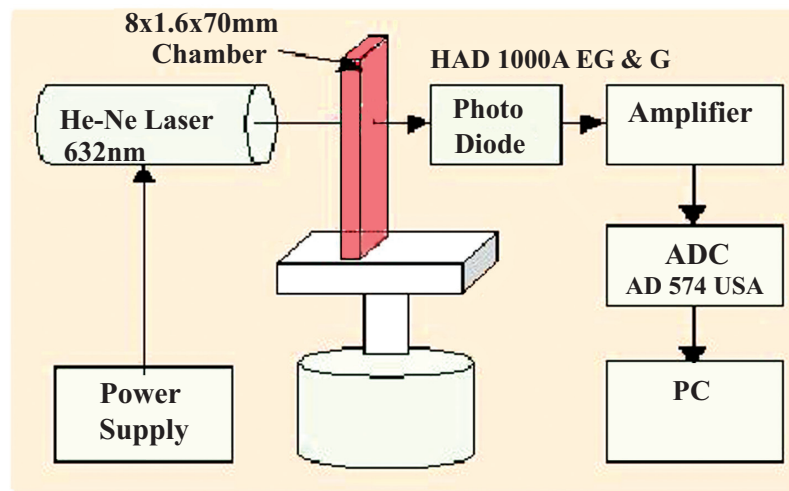

Fig. (1). Schematic diagram of He-Ne laser aggregometer.
Effective number of cells (ENC) represents number of cells in the observed volume (OV) at any given time. Effective cellular sedimentation duration (ECSD) is an indicative of the effective time duration for the formed aggregates to sediment through observed volume.

\subsection{Sample Preparation}

Fresh blood samples were collected in the morning before food, from healthy subjects with glucose concentrations 90 to $120 \mathrm{mg} \%(\mathrm{n}=10)$, diabetes mellitus patients with glucose concentrations 210 to $260 \mathrm{mg} \%$ (glycosylated hemoglobin 9 to $10 \%$ ) with normal cholesterol 130 to 230 $\mathrm{mg} / \mathrm{dl}$ (Group 1, $\mathrm{n}=24$ ) and diabetes mellitus with same glucose level as group1 with hyper cholesterol of more than $280 \mathrm{mg} / \mathrm{dl}$ (Group2, $\mathrm{n}=21$ ) by venepuncture in test tube containing citrate phosphate dextrose (10:1.4), as an anticoagulant. Each blood sample was centrifuged at $2000 \mathrm{~g}$ for $30 \mathrm{~min}$. The supernatant plasma was separated and the buffy coat on the top of cells was separated and discarded. The suspensions of 5\% hematocrit were prepared in plasma. Healthy subjects selected for the study had no clinical disease and their plasma and serum bio- chemical levels are with in normal range. Their systolic and diastolic blood pressures were within normal range and hematocrit within 36 to $45 \%$. The age of healthy and diabetic subjects ranged from 30-60 years and the duration of the disease for diabetes is more than five years. These measurements were carried out in a room maintained at temperature $25 \pm 1$ Centigrade. The statistical analysis of the data was carried out by Student t -test.

\section{RESULTS}

Fig. (2) shows variation of transmitted intensity obtained during aggregation process. Initially there is no significant change in TI for nearly 5 minutes. After that there is gradual increase of TI with large fluctuations in amplitude and attains a maximum value. This is because large number of erythrocytes and their formed aggregates in the observed volume makes large scattering of light and hence minimum transmitted intensity. The amplitude of the fluctuations varies, indicating the change in aggregate size that crossed the observed volume due to gravity. Finally, due to sedimentation of formed aggregates through the observed volume no fluctuations in the transmitted intensity were found, indicating that all the formed aggregates have crossed the observed volume and shows a steady constant value in TI.

Fig. (3) shows the variation of transmitted intensity (TI) of erythrocyte suspension of a normal, group1 and group2 samples. The transmitted intensity reached the steady constant value fastest in group 2 and faster in group 1 comparing to normal.

Fig. (4a) shows change in ASI of four categories of normal subjects in four minutes interval. Initially large numbers of aggregates are found in each category of first 4 minutes interval, and gradually decrease in each category with respect to next 4 minutes intervals of time and so on for normal samples. The pattern of aggregate size index is altered in group1 (Fig. 4b) and group2 (Fig. 4c) of diabetes samples. The sedimentation pattern of different size aggregates varies. Aggregates of various sizes in normal 


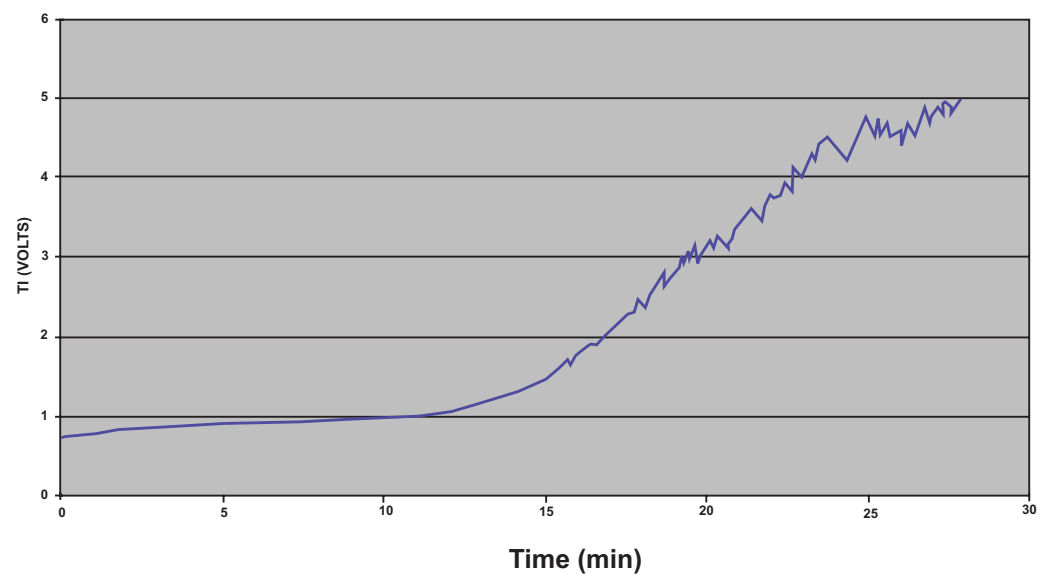

Fig. (2). Variation in transmitted intensity during aggregation process.

samples sediment slowly but these are faster in group1 and fastest in group2 due to the formation of larger size aggregates.

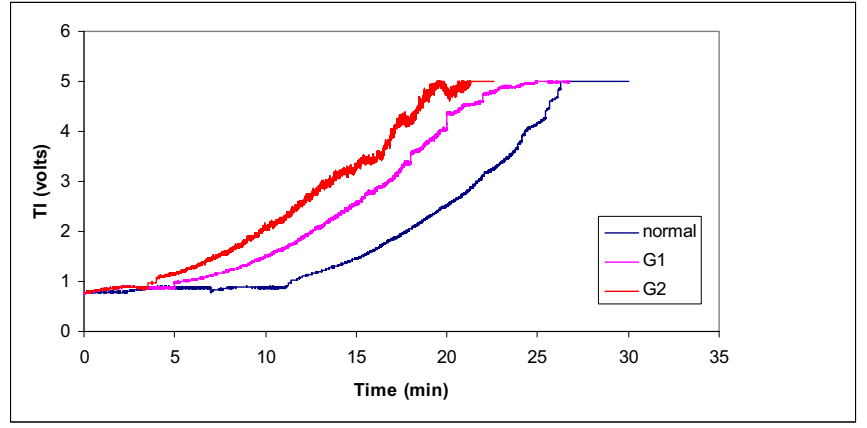

Fig. (3). Variation of transmitted intensity (TI) in normal, group1 and group2 subjects, with respect to time.
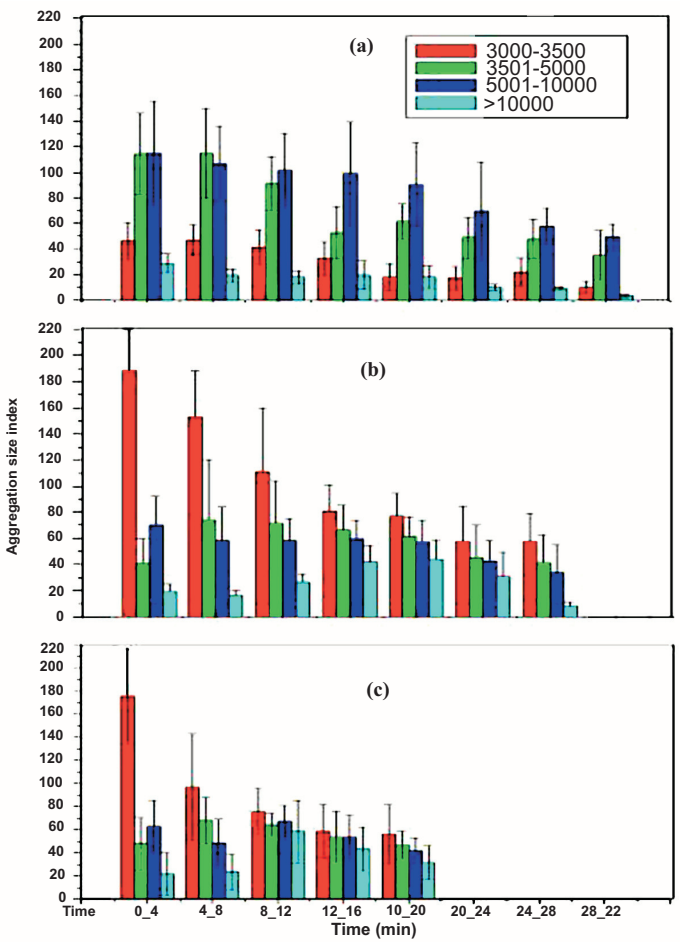

Fig. (4). Comparison of aggregation size index (ASI) of normal (a), group1 (b) and group2 (c) subjects.
Fig. (5) shows a comparison of the effective number of cells $(\mathrm{ENC})$ in observed volume (OV) at various intervals of time in the path of the laser beam. Initially due to well-mixed sample having same hematocrit in observed volume, the value of ENC is same for normal, group1 and group2 of diabetes mellitus. As time increases, the ENC of normal is more than that of other groups throughout the sedimentation process. This is due to large aggregates forms in group2 sediment passed through the observed volume quicker than group1 and control.

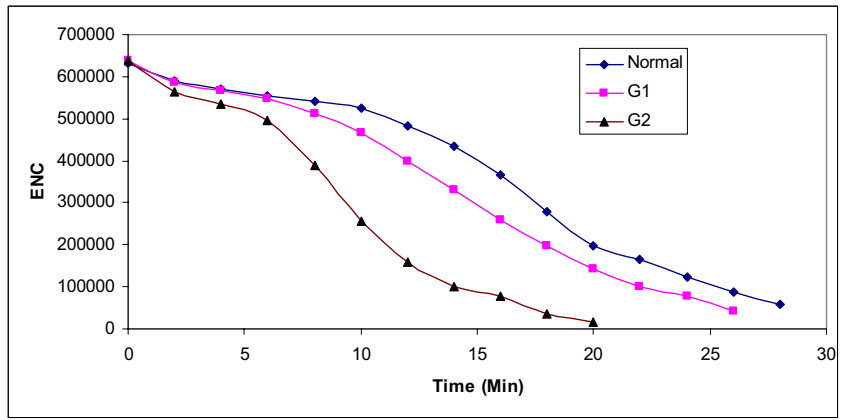

Fig. (5). Comparison of effective number of cells (ENC) of normal, group1 and group2 subjects.

Fig. (6) shows the variation of the effective cellular sedimentation duration (ECSD) of the normal, group1 and group2. This data is an indicator of effective time taken by the aggregates to pass through the observed volume. The aggregates of normal samples take longer duration to pass through the observed volume comparing group1 and group2. The ECSD in group1 is less than normal indicates the formed aggregates in group1 sediments quicker than normal due to influence of glucose, and ECSD in group2 have further less value than group1 due to combined effect of cholesterol and glucose.

Table 1 shows the comparison of the aggregation parameters PIT, PCT, ENC and ECSD. The values of above aggregation parameters are decreased in group1 and further decreased in group2 compared to control, indicating more aggregation in group2 than group1 comparing to control subjects. The data's of ENC and ECSD are chosen corresponding to $14^{\text {th }}$ minute. The elevated plasma fibrinogen in group 1 and further elevated in group2 supports the enhanced aggregation. 


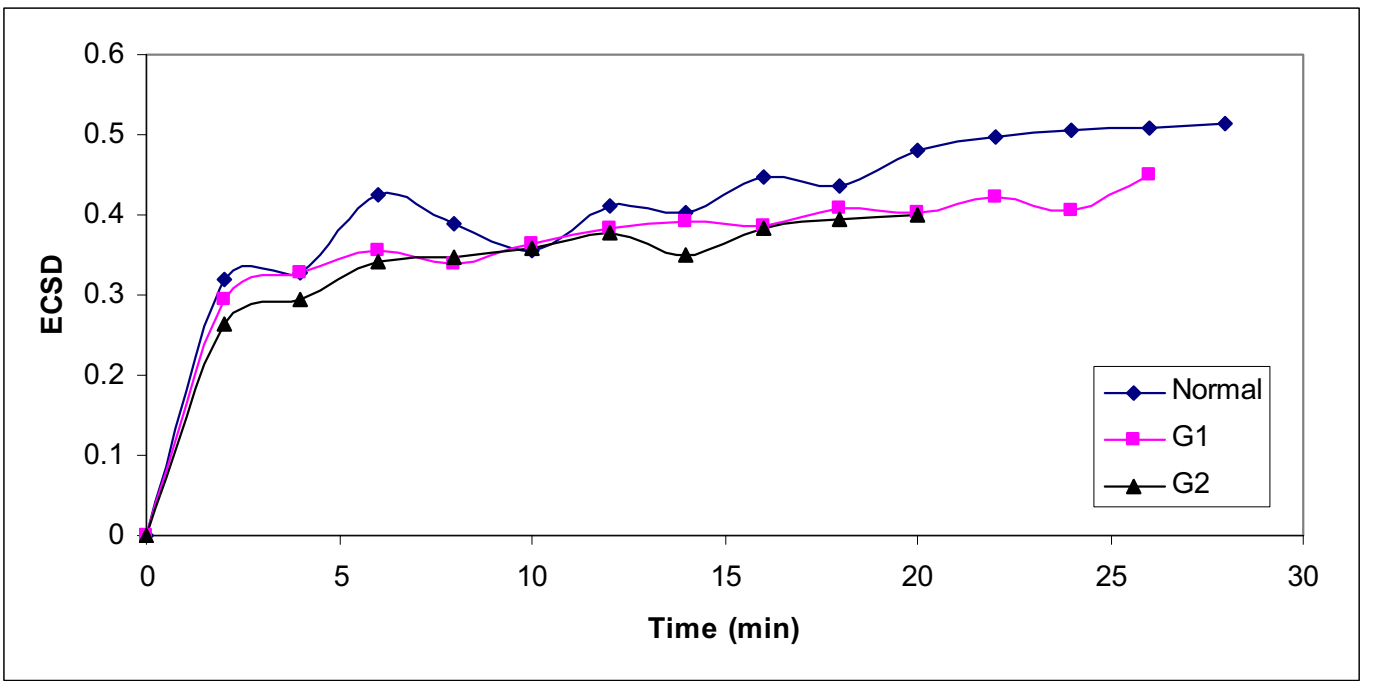

Fig. (6). Comparison of effective cellular sedimentation duration (ECSD) of normal, group1 and group2 subjects.

\section{DISCUSSION}

Aggregation of erythrocytes of control and hyperglycemic subjects with normal and hyper cholesterol were analyzed by online aggregometer. The significant elevation of aggregation of erythrocytes was found in diabetes with hyper cholesterol than diabetes with normal cholesterol compared to control subjects. The extent of RBC aggregation is determined by opposing forces: the repulsive force between the negatively charged cells, the cell-to-cell adhesion induced by plasma proteins [24]. The process of aggregation of erythrocytes is due to presence of plasma protein such as fibrinogen and it was elevated in diabetes mellitus due to hyperglycemia [25] as well as hyper cholesterol [15], which leads to hyper-aggregation. Many studies have reported the elevation of fibrinogen in diabetes as well as in hyper cholesterol subjects. The increase in aggregation in group2 than group1 in this study shows the elevation of fibrinogen, due to the combined effect of hyperglycemic and hyper cholesterol, compared to individual fibrinogen concentrations in hyperglycemic and hyper cholesterol subjects.

The availability of enhanced concentration of heptaglobin further mediates this process. Erythrocyte membrane surface charge and membrane mechanical behavior are the most commonly considered biophysical properties of RBC vis-à-vis their aggregation behavior [26, 27]. The cause of increased in aggregation is due to decrease in sialic acid [28] content of glycoporin A in diabetic's erythrocyte membrane, alteration in the membrane lipid composition and increase in glycosylated hemoglobin (HbA1c). These changes will be common for both hyperglycemia and hypercholesterolemia. Such cells due to their altered properties may not able to carry out tank-tread motion while flowing through micro vessels [29].

Our observations on increased sedimentation of erythrocytes in diabetes with hyper cholesterol supports the formation of large aggregates as these settle down faster in plasma compared to that of normal cholesterol and control subjects. The decrease in effective number of cells (ENC) in observed volume, and decrease in process completion time (PCT) supports this statement. This present work supports the significant elevation of aggregation of erythrocytes in diabetes mellitus with hyper cholesterol due to influence of cholesterol [15].

In conclusion the hyperglycemia and hypercholesterolemia produces significant changes in erythrocytes. These factors combined with increased tendency of adhesiveness and elevated levels of fibrinogen lead to increased erythrocytes aggregation. This elevated aggregation is due to additional effect of hypercholesterolemia in hyperglycemic subjects. This contributory factor in microcirculatory complications as observed in diabetic patients is more severe in presence of hyper-cholesterol.

\section{ACKNOWLEDGEMENT}

The author wishes to thank Prof. Megha Singh (Retired), Biomedical engineering division, Indian Institute of Technology, Madras, who initiates research guidance in

Table 1. Comparison of Aggregation Parameters (Mean \pm SD) of Normal, Group1 and Group2 Subjects

\begin{tabular}{|c|c|c|c|c|c|}
\hline Erythrocytes & $\begin{array}{c}\text { PIT } \\
(\mathbf{m i n})\end{array}$ & $\begin{array}{c}\text { PCT } \\
(\mathbf{m i n})\end{array}$ & $\begin{array}{c}\text { ENC } \\
(\mathbf{1 4 t h} \text { min) }\end{array}$ & $\begin{array}{c}\text { ECSD } \\
(\mathbf{1 4 t h} \text { min) } \\
(\mathbf{s e c})\end{array}$ \\
\hline \hline Normal & $1.25 \pm 0.23$ & $27.25 \pm 5.03$ & $434200 \pm 80600$ & $0.4034 \pm 0.09$ & $272 \pm 5.5$ \\
\hline Group 1 & $1.18 \pm 0.26^{*}$ & $24.08 \pm 3.02^{*}$ & $331500 \pm 12500^{\wedge}$ & $0.3917 \pm 0.08^{\wedge}$ & $406 \pm 13^{* *}$ \\
\hline Group 2 & $1.02 \pm 0.24^{*}$ & $16.23 \pm 2.4^{* *}$ & $101900 \pm 6300^{* *}$ & $0.3502 \pm 0.04 *$ & $504 \pm 21^{* *}$ \\
\hline
\end{tabular}

Compared with the normal, ${ }^{*} \mathrm{p}<0.05$; $^{\wedge} \mathrm{p}<0.005 ;{ }^{* *} \mathrm{p}<0.0004$ 
hemorheology and Dr.Sanjay jayavant for his support in software development for aggregation parameters.

\section{REFERENCES}

[1] Stoltz, J.F.; Donner, M. Clin. Hemorheol. Microrcir., 1987, 7(1), 15.

[2] Rampling, M.W. Cardiovascular flow modeling and measurement with application to clinical medicine, Clarendon Press: Oxford, 1999.

[3] Rampling, M.W.; Meiselman, H.J.; Neu, B.; Baskurt, O.K. Biorheology, 2004, 41, 91.

[4] Stanescu, M.; Zamfirescu, G.; Iordachescu, D. Rom. J. Biophys., 2002, 12,117 .

[5] Mcmillan, D.E. Vasc. Med., 1997, 2, 132.

[6] Wali, R.K.; Jaffe, S.; Kumar, D.; Kalra, V.K. Diabetes, 1988, 37, 104.

[7] Ziegler, O.; Ccandilors, H.; Derriennic, X.S.; Mullar, S.; Donner, M.; Stoltz, J.F.; Drouin, P. Clin. Hemorheol., 1992, 12, 873.

[8] Gandhi, C.R.; Chodbury, D.R. Ind. J. Exp. Biol., 1979; 17: 585.

[9] Vaya, A.; Martinez, M.; Sloves, P.; Barbera, J.L.; Aznar, J. Clin. Hemorheol., 1996, 16, 515.

[10] Smith J.E. Vet. Pathol., 1987; 24: 471.

[11] Babu, N.; Singh, M. Clin. Hemorheol. Microcirc., 2005, 32, 269.

[12] Babu, N.; Singh, M. Clin. Hemorheol. Microcirc., 2004, 31, 273

[13] Shin, S.; Ku, Y.; Babu, N.; Singh, M. Ind. J. Exp. Biol., 2007, 45, 121.

Received: November 07, 2008

Revised: January 20, 2009

Accepted: January 20, 2009

() N. Babu; Licensee Bentham Open.

This is an open access article licensed under the terms of the Creative Commons Attribution Non-Commercial License (http://creativecommons.org/licenses/by-nc/3.0/) which permits unrestricted, non-commercial use, distribution and reproduction in any medium, provided the work is properly cited. 